\title{
Coating PAMAM dendrimer onto PDMS microfluidic chip to resist nonspecific Biomolecules adsorption
}

\author{
Dong Lin ${ }^{1, a^{*}}$, Qin Zhao ${ }^{2, b}$, Miaomiao Yan ${ }^{1, \mathrm{c}}$, Giuge Hou ${ }^{1, \mathrm{~d}}$ \\ ${ }^{1}$ School of Pharmacy, Binzhou Medical University, Yantai, 264003, China \\ ${ }^{2}$ College of Food Engineering, Ludong University, Yantai 264025, China \\ alindong3503@163.com, bcandyffff@163.com, '342302878@qq.com, dguigehou@163.com.
}

\begin{abstract}
Keywords: PAMAM dendrimer; microfluidic chip; caoting; hydrophilicity; adsorption
\end{abstract}
Abstract. The surface of poly(dimethylsiloxane)(PDMS) microfluidic chips were modified using polyamidoamine (PAMAM) dendrimer via physical coating. The results showed that the surface of the modified chips was coated by a dense, uniform, continuous, hydrophilicity layer of PAMAM. The hydrophilic of the chip surface was markedly improved. The contact angle of the chips modified decreased from $108.9^{\circ}$ to $46.1^{\circ}$. Adenosine and L-lysine were separated via the modified PDMS microfluidic chips. Compared with unmodified chips, the modified chips successfully separated the two biomolecules. The separation efficiencies of adenosine and L-lysine were $7.19 \times 10^{4}$ Plates $/ \mathrm{m}$ and $8.111 \times 10^{4}$ Plates $/ \mathrm{m}$ respectively, and the resolutions $\left(\mathrm{R}_{\mathrm{S}}\right)$ was 4.03 . This research may provide a new and effective method to resist nonspecific adsorptionon the PDMS surface and the application of PDMS microfluidic chips in the determination of trace biomolecules.

\section{Introduction}

PDMS microfluidic chips are grasping more and more strengths with advantages such as easy fabrication, cheap costs, massive production, better transmittance and dielectricity etc ${ }^{[1,2]}$. However, PDMS microfluidic chips also exists some defects: like other polymers, its surface has a strong hydrophobicity. And many biomolecules will be forcefully absorbed onto microchannel surface because of the hydrophobic interaction ${ }^{[3]}$. This may lead to a distorted analysis result and a lower separation efficiency. Therefore, it's necessary to modify the surface of PDMS microfluidic chips for improving its hydrophilicity and inhibiting the absorption between biomolecules and the microchannel surface.

Presently, surface modification of PMMA microfluidic chips is only limited to using traditional linear polymers ${ }^{[4,5]}$ and there are many problems without resolution, such as coating difficulties due to rapidly increasing viscosity corresponding to a higher solution concentration and poor reproducibility due to inadequate functional groups to bond on the inner surface of the chips.

Based on above issues, this research initially adopts PAMAM dendrimer via physical coating to modify the surface of PDMS microfluidic chips. Compared to traditional linear polymer, the dendrimers has some unique features such as three-dimensional morphology, , higher solubility, lower viscosity, more reactive end groups, which makes it a very potential material for modification.

\section{Experimental}

\section{Materials and equipment}

PDMS microfluidic chips were obtained using silicon model via copy pressed film ${ }^{[6]}$. PAMAM dendrimers of different generations were fabricated by divergent method ${ }^{[7]}$, and products of the second, third, fourth generation being marked G2, G3, G4 respectively.

L-lysine, adenosine(AR , Sigma company, USA); dimethylsulfoxide, p-toluenesulfonic(AR, Da Mao Chemical Reagent Company, Tianjin, CHN); sodium dihydrogen phosphate, disodium hydrogen phosphate(AR, Guang Cheng Chemical Reagent Company, Tianjin, CHN); methanol(LC, Shang Hai 
Chemical Reagent Company, Shanhai, CHN); sodium hydroxide(AR, Lai Yang Kangde Chemical Industry Company, Yantai, CHN); the water used in this experiment is double-deionized.

S-2500 scanning electron microscope(SEM): Japan Hitachi Company; XTB-1 stereoscope: Nanjing Jiangnan Electro-optic Company; DSA100 contact angle meter: German KRUSS Company; Microfluidic chip testing and analysis device: self-made.

\section{Process of PDMS microfluidic chip modification}

\section{(1) Coating solution preparation}

The synthesized dendritic PAMAM G2, G3, G4 were dissolved respectively in the methanol getting the coating solution with a mass fraction of $10 \%$. Then the solution was reserved to use after being filtered using $45 \mu \mathrm{m}$ cellulose filters.

\section{(2) PDMS microfluidic chips pretreating}

PDMS microfluidic chips were rinsed using $0.5 \mathrm{~mol} / \mathrm{L} \mathrm{NaOH}$ solution at a nitrogen pressure of $0.3 \mathrm{MPa}$ for $1 \mathrm{~h}$, and then using double-deionized water for $30 \mathrm{~min}$, and then using methanol for $30 \mathrm{~min}$. Finally dry nitrogen was used to blow-dry the chips.

\section{(3) Coating chips fabrication}

Three dendritic PAMAM methanol solutions with a mass fraction of $10 \%$ were pressed respectively into pretreated chips and kept a slow flow rate for $15 \mathrm{~min}$ so that the coating solution would have a full contact with the inner wall of chips. Then nitrogen was used to blow off residual solutions in a slow rate. After that, the chips coated were put into the oven with an adequate stream of nitrogen surrounded at $40 \square$ for $3 \mathrm{~h}$. The product was PDMS microfluidic chip by virtue of physical coating modification.

\section{Results and discussion}

\section{Contact angle measurements}

The contact angle was the angle $\theta$, measured through the liquid, where a liquid/vapor interface meets a solid surface. It quantified the wettability of a solid surface. The smaller the contact angle was, the better its hydrophilicity was. In order to show the hydrophilic difference between modified PDMS microfluidic chips and unmodified ones, their contact angles were measured using contact angle meter. Results were listed in Table 1.

Table 1 Contact angle of PDMS microfluidic chips

\begin{tabular}{ccccc}
\hline sample & unmodified chips & G2 modified chips & G3 modified chips & G4 modified chips \\
\hline$\theta$ & $108.9^{\circ}$ & $67.8^{\circ}$ & $52.4^{\circ}$ & $46.1^{\circ}$ \\
\hline
\end{tabular}

It was showed that the contact angle of PDMS microfluidic chips unmodified was $108.9^{\circ}$. But after dendritic PAMAM modification, the contact angle decreased considerably, especially for chips from G4 whose contact angle decreased to 46.1, which indicated that dendritic PAMAM modification effectively overcame the hydrophobicity of surface of PDMS microfluidic chips.

It was also found that along with the increase of generations of PAMAM, the decreased rate of contact angle expanded, for with the increase of generations, molecule weight expanded, three-dimensional spherical area enlarged, the number of hydroxyl groups which were on the surface of molecules and interacted with the inner wall of chips and the number of ester groups and amide groups multiplied, the active sites with inner wall of chips further increased. As a result, the thickness of hydrophilic coat augmented and its hydrophilicity became better.

\section{Electroosmotic flow measurements}

With dimethylsulfoxide solution as the neutral maker, the electroosmotic flows of unmodified chips, G2 chips, G3 chips and G4 chips were measured in different $\mathrm{pH}$ conditions, and results were showed in Figure 1. In the range of $\mathrm{pH} 3.0-9.0$, along with the increase of $\mathrm{pH}$, electroosmotic flow of unmodified chips and modified chips increased but the electroosmotic flow of modified chips was much lower than that of unmodified, which expressed that dendritic PAMAM coat could effectively shield the 
dissolution of electriferous groups on the inner wall of chips. Then the charge density on the inner wall of chips decreased, and the electroosmotic flow decreased dramatically.

Figure 2 also told that along with the adding of generations of dendritic PAMAM, the decreased rate of electroosmotic flow increased, for as generations added, their molecules increased, the number of active groups interacting between molecules and the inner wall of chips augmented, all of which made the thickness of hydrophilic coat increased so that groups such as hydroxyl were shielded from dissolution. And thus the electroosmotic flow further decreased.

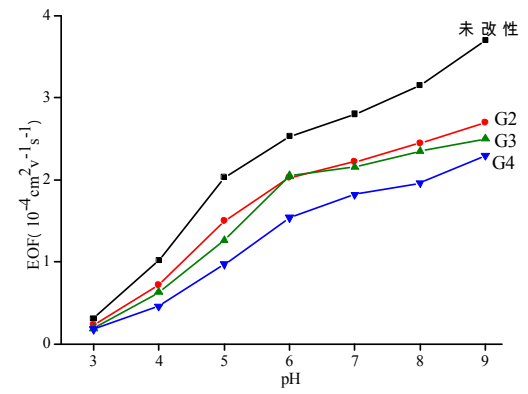

Figure 1 Effect of the buffer $\mathrm{pH}$ on EOF in chips modified using physical coating

\section{Separation detection on L-lysine and adenosine}

L-lysine and adenosine were conducted a separation detection using PDMS microfluidic chips via physical coating from G2, G3 and G4 with 40mmol/L phosphate buffer(pH4.8), 500V/cm separation field and $214 \mathrm{~nm}$ detection wavelength. Results were showed in Figure 2 . At the same time, the separation efficiency and Rs of modified chips were showed in Table 2.

The result showed adenosine and L-lysine were not separated effectively using unmodified PDMS microfluidic chips with a wider detect peak, a worst peak pattern and a tailing peak. The reason was that the hydrophobic effect between biomolecules and the inner wall of chips produced a heavy absorption; while PDMS microfluidic chips modified by dendritic PAMAM generated a dense layer of hydrophilic coat, and this coat effectively blocked the absorption from the inner wall of chips to biomolecules, thus a successful separation for adenosine and L-lysine was realized. The detect peak was incisive and the separation clear. Figure 3 also told that along with the adding of generations, the migration time of components extended, for as generation added, the coat formed became more dense, the electroosmotic flow decreased. These further led to a lower migration rate. Tab. 5.1 showed that, compared to unmodified chips, the separation efficiency and $R_{s}$ of modified chips in G2, G3 and G4 increased dramatically, especially for chips in G4, whose separation efficiency increased more evidently. Efficiencies as many as $7.19 \times 10^{4}$ plates $/ \mathrm{m}$ for adenosine, and $8.11 \times 10^{4} \mathrm{plates} / \mathrm{m}$ for L-lysine were achieved. And separation degree was 4.03 .

The comparative results of separation detection on two molecules using modified chips from G2, G3 and G4 showed that as the generations of PAMAM added, their separation peak patterns were better, and the separation efficiency and separation degree increased. That was because as the generations added, the number of molecules rose, and the thickness of coat on the inner wall of chips increased, so that the hydrophilicity of the surface of chips increased more effectively, thus the absorptive effect for two molecules was blocked and a better separation was obtained. 


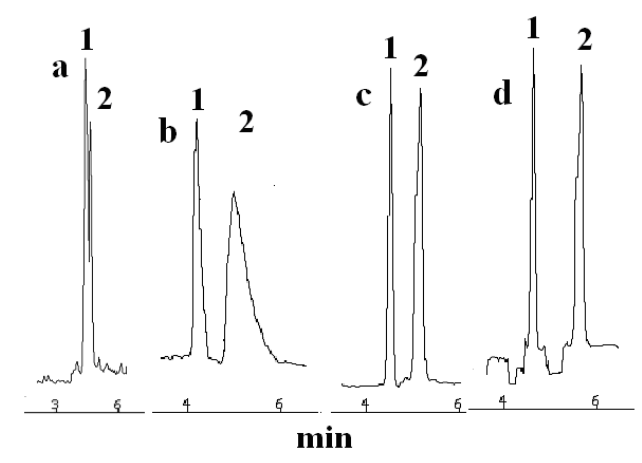

Figure 2 Electrophoresis figure of PDMS microfluidic chips modified by hyperbranched polyamide ester using physical coating. 1 adenosine; 2 L-lysine; (a) unmodified chips; (b) G2 modified chips; (c) G3 modified chips; (d) G4 modified chips

Table 2 Separation efficiency and Rs of unmodified and modified chips

\begin{tabular}{cccc}
\hline \multirow{2}{*}{$\begin{array}{c}\text { PDMS microfluidic } \\
\text { chips }\end{array}$} & \multicolumn{2}{c}{ Separation efficiencyN $\times 10^{4}($ Plates $/ \mathrm{m})$} & \\
\cline { 2 - 3 } & adenosine & L-lysine & $\mathrm{R}_{\mathrm{s}}$ \\
\hline unmodified & 0.87 & 1.26 & 0.24 \\
G2 modified & 2.03 & 2.36 & 0.91 \\
G3 modified & 3.28 & 6.47 & 2.63 \\
G4 modified & 7.19 & 8.11 & 4.03 \\
\hline
\end{tabular}

\section{Conclusion}

The surface of ploy(dimethylsiloxane)(PDMS) microfluidic chips were modified using polyamidoamine dendrimer via physical coating. The surface formed a dense, uniform and continuous hydrophilic coat. The hydrophilicity improved a lotd and effectively blocked the absorption on biomolecules.

\section{Acknowledgements}

This work was funded by Shandong Provincial Natural Science Foundation, China (No. ZR2014BP016, ZR2015PC002), and A Project of Shandong Province Higher Educational Science and Technology Program (No. J15LC01).

\section{References}

[1] Y. W. Lin, M. F. Huang, H. F. Chang. Nanomaterials and chipbased nanostructures for capillary eletrophoretic separations of DNA.[J]. Eletrophoresis, 2005, 26(2): 320-328.

[2] O. Hofman, D. Che, K. A. Cruickshank, et al. Adaptation of capillary isoelectric focusing to mictochannels on a glass chip [J]. Anal Chem, 1999, 71: 678-683..

[3] D. Bodas, J.Y. Rauch, C. K. Malek. Surface modification and aging studies of addition-curing silicone rubbers by oxygen plasma. European Polymer Journal, 2008, 44: 2130-2139.

[4] A. J. Wang, J. J. Xu, H. Y. Chen. Nonionic surfactant dynamic coating of poly(dimethylsiloxane) channel surface for microchip electrophoresis of amino acids. Anal Chim Acta, 2006, 69: 188-194. [5] S. Hu, X. Ren, M. Bachman, C. E. Sims, G. P. Li, Allbritton N. Surface modification of poly(dimethylsiloxane) microfluidic devicesby ultraviolet polymer grafting. Anal Chem, 2002, 74: 4117-4123. 
[6]M. Y. Ye, Q. Fang, X. Z. L. F. Yin, Fang. Studies on bonding techniques for poly( dimethylsiloxane) microf luidic chips.Chem. J. Chinese Universities, 2002,23(12):2243-2246. [7] D. Lin, Q. Zhao, J. T, Han, G. G. Hou, S. N. Zheng. Research on complexation between PAMAM dendrimer with a tri(2-aminoethyl)amine Core and $\mathrm{Zn}^{2+}$. Chinese Journal of Instrumental Analysis 